\title{
COMMENT
}

\section{Improving pharmaceutical trials for children: a call to the pediatric academic community}

\author{
Scott C. Denne ${ }^{1}$ and Jonathan M. Davis ${ }^{2}$ \\ Pediatric Research (2018) 84:319-320; https://doi.org/10.1038/s41390-018-0101-1
}

Pediatricians have long advocated that high-quality clinical trials must be conducted to inform proper use of medications in children. Since the passage of the Best Pharmaceuticals Act for Children (BPCA), the Pediatric Research Equity Act (PREA), and the Food and Drug Administration Safety and Innovation Act (FDASIA), significant progress has been made. Since 2007, over 400 pediatric studies have been conducted and nearly 500 pediatric labels have been changed. ${ }^{1}$ Nevertheless, there remain ongoing challenges in the effort to conduct timely and efficient pediatric trials using achievable and appropriate clinical endpoints.

In this issue of Pediatric Research, Kelly et al. $^{2}$ highlight the paucity of validated pharmacodynamic (PD) endpoints in children; identifying validated, age-appropriate, conditionspecific pediatric efficacy, and safety PD endpoints is a serious challenge currently faced by pediatricians, scientists, drug developers, and regulatory agencies. These endpoints need to be clinically relevant, responsive to both treatment and/or disease progression, and be reproducible and reliable. Development of validated PD endpoints has great potential to increase the speed and efficacy of pediatric trials. However, creating these PD endpoints will be a painstaking process, as development must occur across multiple developmental stages (preterm infants through adolescents), and relevant to most pediatric diseases. This is a worthy and important effort which should be supported by the entire pediatric academic community. This will also require cooperation and coordination of the international regulatory agencies, the pharmaceutical industry, parents, and children. As pointed out by Kelly et al. ${ }^{2}$, developing these endpoints can be incorporated into many if not all pediatric trials, but will require consultation, coordination, and planning early in the clinical trial design process.

While we await the development of validated PD endpoints, pediatric pharmaceutical trials will continue to be conducted. Appropriate, achievable, and clinically meaningful endpoints for these trials remain a challenge for the FDA and the pharmaceutical industry. Even large pharmaceutical companies have a limited number of highly qualified pediatric experts; this is also true of the FDA and other international regulatory agencies. This is especially important since a significant proportion of pediatric trials fail because of poorly chosen clinical endpoints. ${ }^{3}$ It is imperative that the FDA and industry representatives consult more extensively with pediatric experts early in the clinical trial design process. Grants from the FDA to the Institute for Advanced Clinical Trials for Children (IACT) and Duke University may help to provide some additional expertise in selecting clinical endpoints. ${ }^{4}$ However, it will be important to engage the wider pediatric academic community in this effort and pediatric academicians must be eager and willing to participate.

In addition to clinical endpoints, another significant challenge for pediatric drug trials is the need for many sites (often in multiple countries) in order to recruit sufficient subjects. These multisite trials are often constructed de novo for each new pediatric trial, adding complexity, cost, and time for study completion. Moreover, it is common to find that a significant percentage of sites engaged in a pediatric trial never recruit a single subject. Fortunately, two new networks have been created to serve as a multisite, multinational platform for pharmaceutical trials: one in Europe (European Clinical Trials for Children Consortium) and one in the US (IACT)., ${ }^{5,6}$ These two new networks are highly promising initiatives to increase the efficiency of conducting pediatric drug trials in multiple countries simultaneously. However, the pharmaceutical industry must begin to place their trials in these networks so they can be continuously refined and improved. Similarly, since the legislation is facilitating the conduct of more clinical trials, academic sites must be willing to participate in these networks and conduct high-quality studies in a highly efficient manner.

We all share the goal of bringing effective new therapies to children as quickly as possible. Improvements in speed and efficiency will require developing and validating PD endpoints, choosing more robust clinical endpoints, and engaging multiple sites in multiple countries simultaneously. Although the involvement of the pediatric academic community is crucial to these efforts, academic investigators have not always been highly supportive of these activities. While this has been slowly changing for the better, there is still progress that needs to be made. This includes training a sufficient number of pediatric trialists across multiple subspecialties (e.g., workforce development), valuing and rewarding the contributions of faculty members who conduct pediatric multicenter pharmaceutical trials, and ensuring an efficient contract and institutional review process. By partnering with industry and the regulatory agencies, the pediatric academic community's wealth of talent, expertise, and commitment can greatly improve the process of bringing new drugs and devices to children.

Publisher's note: Springer Nature remains neutral with regard to jurisdictional claims in published maps and institutional affiliations.

${ }^{1}$ Department of Pediatrics, Indiana University, Indianapolis, IN, USA and ${ }^{2}$ Department of Pediatrics and the Tufts Clinical and Translational Research Institute, Tufts University, Boston, MA, USA

Correspondence: Scott C. Denne (sdenne@iu.edu)

On behalf of the Pediatric Policy Council.

Published online: 9 July 2018 
Improving pharmaceutical trials for children: a call to the pediatric... SC Denne and JM Davis

320

\section{REFERENCES}

1. U.S. Food and Drug Administration. Pediatric product development. https://www. fda.gov/Drugs/DevelopmentApprovalProcess/DevelopmentResources/ ucm049867.htm. Accessed 4 June 2018.

2. Kelly, L. E., Sinha, Y., Barker, C. I. S., Standing, J. F. \& Offringa, M. Useful pharmacodynamic endpoints in children: selection, measurement and next steps. Pediatr. Res. (2018). https://doi.org/10.1038/pr.2018.38.

3. Momper, J. D., Mulugeta, Y. \& Burckart, G. J. Failed pediatric drug development trials. Clin. Pharmacol. Ther. 98, 245-251 (2015).
4. FDA Awards Funding to Support Pediatric Clinical Trials Research. https://blogs.fda. gov/fdavoice/index.php/2017/11/fda-awards-funding-to-support-pediatricclinical-trials-research/. Accessed 4 June 2018.

5. Launch of a new public-private partnership to improve clinical trial infrastructure to facilitate the development of new drugs for children in Europe. http:// conect4children.org/news/press-release-launch-of-the-c4c-project/. Accessed 4 June 2018.

6. Institute For Advanced Clinical Trials For Children. https://www.iactc.org. Accessed 4 June 2018 